\title{
On the Consistency Analyzing of A-SLAM for UAV Navigating in GNSS Denied Environment
}

\author{
A. Ersan Oguz*, Hakan Temeltas** \\ *Electronics Engineering Department, Turkish Air Force Academy, Yesilyurt, \\ 34149 Istanbul, Turkey (e-mail: ae.oguz@hho.edu.tr) \\ **Control Engineering Department, Istanbul Technical University, 34469 \\ Istanbul, Turkey (e-mail: hakan.temeltas@elk.itu.edu.tr)
}

\begin{abstract}
In this paper, EKF Based A-SLAM concept is discussed in detail by presenting the formulas and MATLAB Simulink model, along with results. The UAV kinematic model and state-observation models for the EKF Based A-SLAM are developed to analyze the consistency. The covariance value caused by the EKF structure is analyzed. This value was calculated by filtering with error between the UAV's actual and estimated positions. It is concluded that the calculated covariance value diminishes despite error and does not decrease as a result of the inconsistency of the EKF Based A-SLAM structure. The necessary conditions for the consistency of the EKF Based A-SLAM structure are described and the consistency of the EKF Based A-SLAM is consequently investigated by considering these conditions for the first time by using simulation results. The analysis during this landmark observation exhibits that a jagged UAV's trajectory indicates an inconsistency caused by the EKF Based A-SLAM structure with the provocation of error accumulation. Finally, the major reasons of the filter inconsistency can be listed as unobservable subspace and Jacobian matrices used for linearization. As a future work, the methods that can be used to provide consistency of the EKF Based A-SLAM are proposed for better UAV navigation performance.
\end{abstract}

\section{Introduction}

Unmanned Aerial Vehicles (UAVs) have a quite distinguished role for decision makers and operational agents due to their extensive usage and large variety of applications, such as reconnaissance and surveillance in the military and civilian areas. The main usage purpose of UAVs is autonomous navigation that provides flexible functionality particularly on a stand-alone flight in autonomous operations. It is crucial to determine the UAVs precise position for better navigation. Global Navigation Satellite System (GNSS), which is actually 
preferred to geolocation tools in the world, is also the most adequate tools for the UAV position determination process. However, it can be hard to have successful results for this process in GNSS denied environments. Thus, there exist a great deal of work in the literature about this problem. In GNSS denied environments, when the map knowledge also does not exist, the problem of simultaneous position determination and production of map information can be solved by Extended Kalman Filter (EKF) Based Airborne Simultaneous Localization And Mapping (A-SLAM), which is the consistency analysis issue in this paper.

The SLAM problem is a hot topic for both industrial and military robotic research. The SLAM problem was introduced by R. Smith and P. Cheesman [1], then was detailed by G. Dissanayake, H. F. Durrant-Whyte and T. Bailey [2], and finally was presented as a whole concept by T. Bailey and H. F. Durrant-Whyte [3, 4]. While the researchers were interested in the SLAM problem for territorial, aerial, and marine vehicles, they examined a way to decrease the error that is caused by filter structure. In these studies, the structure of the Kalman filter-based SLAM effects of partial observability [5-7], stability [8] and the consistency problem [9, 10] were examined to present recommendations for solutions to the problem. Airborne SLAM applications are continued simultaneously [11-14]. It was very important to know the current location in the works on autonomous navigation in aircraft $[15,16]$ and the creation of an accurate map. The optimal level of mapping method was also examined in L. M. Paz and J. Neira [17].

In the literature, filter consistency can be studied as a separate title. In the case that the estimated covariance of the filter is greater than or equal to the actual covariance that is the covariance difference between actual and estimated positions of UAVs, the filter is called consistent. The filter consistency is very important in terms of performance. When the filter is inconsistent, error accumulation increases, and consequently the vehicle location is determined with some error. The filter consistency is still an attractive research topic and researchers have made numerous attempts to improve consistency. The consistency conditions of the Kalman filter, named the state estimator, were revealed by Y. Bar-Shalom, X. Rong Li and T. Kirubarajan [18]. T. Vidal-Calleja, J. Andrade-Cetto and A. Sanfeliu demonstrated the marginal stability status of the Kalman filter [8], and J. A. Castellenos, J. Neira and J. D. Tardos analyzed the consistency of the suboptimal filter and examined limits to the SLAM consistency [10]. It is shown by T. Bailey, J. Nieto, J. Guivant, M. Stevens and E. Nebot that in the case of two core symptoms, the EKF-based SLAM filter is inconsistent [9].

However, the inconsistency problem for an aerial vehicle has not been identified yet. SLAM filters that are designed for aerial vehicles must include the kinematic model. In this case, the filter consistency problem becomes more complex. In this work, a SLAM filter designed for aircraft and the consistency problem have been analyzed for the aircraft kinematics model. In the literature, the SLAM problem for an aircraft is known as an A-SLAM. The inconsistency in the EFK-based ASLAM filters is expressed clearly with the proposed analysis methods. 
The aircraft kinematic model is shown in detail in Section 2. The structure of the EKF based A-SLAM is described in Section 3. In the subsections, non-linear prediction and the observation model are demonstrated, the procedures in estimation and correction steps are described and the Jacobian matrices used for linearization are established. The problem of consistency and its symptoms are described in Section 4. Simulations for several scenarios are performed and, according to the simulation results, the consistency structure of the EKF based ASLAM are examined in Section 5. The obtained simulation results were evaluated in Section 6 and finally, the solution methods proposed in future works with emerging inconsistency problems are discussed.

\section{The UAV Kinematical Model}

The transformation of the body frame motions of an aerial vehicle to the navigation frame plays an important role since the global mapping of the environment requires aerial vehicle equation of motion to the global frame. Euler angle transformations can be used for this purpose. The aerial vehicle body frame accelerations of the directional navigation and angular rates are transferred to the navigation frame, and the position of the aerial vehicle is calculated in navigation frame. The general equation generated during this process is expressed as the kinematic equation of the aerial vehicle. The matrix expression obtained by the transfer of directional acceleration to navigation frame of aerial vehicles is

$\left[\begin{array}{c}\dot{x} \\ \dot{y} \\ \dot{z}\end{array}\right]=\left[\begin{array}{ccc}\cos \theta \cos \Psi & \cos \Psi \sin \theta \sin \phi-\cos \phi \sin \Psi & \cos \Psi \sin \theta \cos \phi+\sin \phi \sin \Psi \\ \cos \theta \sin \Psi & \cos \phi \cos \Psi+\sin \theta \sin \phi \sin \Psi & -\sin \phi \cos \Psi+\sin \theta \cos \phi \sin \Psi \\ -\sin \theta & \cos \theta \sin \phi & \cos \theta \cos \phi\end{array}\right] \cdot\left[\begin{array}{l}u \\ v \\ w\end{array}\right]$

The transformation matrix of angular rates from body frame to navigating frame is

$$
\left[\begin{array}{c}
\dot{\phi} \\
\dot{\theta} \\
\dot{\Psi}
\end{array}\right]=\left[\begin{array}{ccc}
1 & \sin \phi \tan \theta & \cos \phi \tan \theta \\
0 & \cos \phi & -\sin \phi \\
0 & \sin \phi \sec \theta & \cos \phi \sec \theta
\end{array}\right] \cdot\left[\begin{array}{c}
p \\
q \\
r
\end{array}\right]
$$

These two matrix equations are referred to as the kinematic model of the aerial vehicle. The vector $[\mathrm{u}, \mathrm{v}, \mathrm{w}]$ is the directional acceleration in the body frame and $[\mathrm{p}, \mathrm{q}, \mathrm{r}]$ is the angular rates of the body frame. 


\section{EKF-based Airborne SLAM}

An EKF-based filter was used for the A-SLAM in this section. First, the nonlinear model of prediction and observation is given and then the estimate and update of the steps of the EKF are described.

\subsection{The Non-Linear Prediction and Observation Model}

The aerial vehicle state vector and map vector come from the state vector used in non-linear system model. The aerial vehicle state vector is composed of the position $(\mathrm{x}, \mathrm{y}, \mathrm{z})$, velocity $(\mathrm{Vx}, \mathrm{Vy}, \mathrm{Vz})$ and the Euler angles (roll, pitch and yaw). The map vector is made up of the position of each landmark $\left(\mathrm{x}_{\mathrm{L}}, \mathrm{y}_{\mathrm{L}}, \mathrm{z}_{\mathrm{L}}\right)$. The length of this vector is $3 * n$ (n: number of landmark).

$x(k)=\left[\begin{array}{l}x_{U A V} \\ x_{M A P}\end{array}\right]$

EKF is a filter structure in a non-linear system that estimates the next step from the previous state and the observation value. As in the Kalman filter, it has estimation and correction steps. The state space model expression that will be used in filters is

$x(k+1)=f(x(k), u(k), v(k))$

In the state space model, the state vector at step of $k+1$ depends on the state vector and input data of step $\mathrm{k}$.

The landmark position can be found as the range, bearing, and elevation as in the observation model. The transformation of that value from sensor frame to navigation frame is performed by the transformation matrix. The observation model depends on the position information of the aerial vehicle at step $\mathrm{k}$. It is stated as

$z(k+1)=h(x(k), \omega(k))$

where $\mathrm{v}(\mathrm{k})$ and $\mathrm{w}(\mathrm{k})$ are zero mean white noise. Their covariance is $Q=\left[v(k) \cdot v(k)^{T}\right]$ and $R=\left[\omega(k) \cdot \omega(k)^{T}\right]$

The noise covariance can be written as

$E[v(k)]=0$

$E\left[v(k) v(k)^{T}\right]=Q(k)$

and

$E[w(k)]=0$ 
$E\left[w(k) w(k)^{T}\right]=R(k)$

In equation (4) the state space model in explicit for can be written as

$$
f_{\text {uav }}=\left[\begin{array}{c}
P^{n}(k) \\
V^{n}(k) \\
\Psi^{n}(k)
\end{array}\right]=\left[\begin{array}{c}
P^{n}(k-1)+\Delta t * V^{n}(k-1) \\
V^{n}(k-1)+\Delta t * T_{b}^{n}(k-1) * f^{b}(k) \\
\Psi^{n}(k-1)+\Delta t * E_{b}^{n}(k-1) * w^{b}(k)
\end{array}\right]+\left[\begin{array}{c}
w_{P^{n}} \\
w_{V^{n}} \\
w_{\Psi^{n}}
\end{array}\right]
$$

Here it will be seen that the state space model is composed of the position, directional velocity and Euler angle. The state space model is obtained from the kinematic equation of the aerial vehicle.

\subsection{EKF Estimation Step}

The A-SLAM needs the stochastic estimation, which is a guess of the UAV position parameter at step $\mathrm{k}$ from the previous state with Gaussian noise. The structure is constructed by the kinematic system model as compatible with the EKF structure. As in the EKF-based A-SLAM it is elementarily composed of estimation and update steps. For the model linearization process, the Jocobian matrices are used. In the EKF-based A-SLAM, the state estimation at step k, due to input variables and the state at step-k, can be written as

$$
\hat{x}(k+1 \mid k)=f(x(k \mid k), u(k))
$$

The observation estimation is

$$
z(k+1 \mid k)=h(\hat{x}(k+1 \mid k))
$$

The estimated covariance calculated by is

$$
P(k+1 \mid k)=\nabla F_{x} \cdot P(k \mid k) \cdot \nabla F_{x}{ }^{T}+\nabla F_{u} \cdot Q \cdot \nabla F_{u}{ }^{T}
$$

\subsection{EKF Update State}

The estimation update step is performed next to the EKF estimation step. The updated state estimation expression is

$x(k+1 \mid k+1)=\hat{x}(k+1 \mid k)+W . v(k+1)$

where $\mathrm{W}$ is the Kalman gain and $v(k+1)$ is innovation. The updated covariance is

$$
P(k+1 \mid k+1)=P(k+1 \mid k)-W \cdot S(k+1) \cdot W^{T}
$$

The calculation of the innovation and its covariance (covariance error) is done by

$$
v(k+1)=z(k+1)-z(k+1 \mid k)
$$




$$
S(k+1)=\nabla H_{x} P(k+1 \mid k) \nabla H_{x}{ }^{T}+R
$$

Finally the Kalman gain is

$$
W=P(k+1 \mid k) . \nabla H_{x}^{T} \cdot S^{-1}(k+1)
$$

The Jacobian matrix $\nabla f_{\text {uav }}(k)$ used for the model linearization in A-SLAM that is composed of partial differentials of position, velocity and the Euler angle of the UAV kinematic model is

$$
\nabla f_{\text {uav }}(k)=\left[\begin{array}{ccc}
\frac{\partial P^{n}(k)}{\partial P^{n}(k-1)} & \frac{\partial P^{n}(k)}{\partial V^{n}(k-1)} & \frac{\partial P^{n}(k)}{\partial \Psi^{n}(k-1)} \\
\frac{\partial V^{n}(k)}{\partial P^{n}(k-1)} & \frac{\partial V^{n}(k)}{\partial V^{n}(k-1)} & \frac{\partial V^{n}(k)}{\partial \Psi^{n}(k-1)} \\
\frac{\partial \Psi^{n}(k)}{\partial P^{n}(k-1)} & \frac{\partial \Psi^{n}(k)}{\partial V^{n}(k-1)} & \frac{\partial \Psi^{n}(k)}{\partial \Psi^{n}(k-1)}
\end{array}\right]
$$

The Jacobian matrix $\nabla f_{w}(k)$ is composed of the differential equation of the nonlinear process gain model input variables is

$$
\nabla f_{w}(k)=\left[\begin{array}{cc}
\frac{\partial P^{n}(k)}{\partial f^{b}(k-1)} & \frac{\partial P^{n}(k)}{\partial w^{b}(k-1)} \\
\frac{\partial V^{n}(k)}{\partial f^{b}(k-1)} & \frac{\partial V^{n}(k)}{\partial w^{b}(k-1)} \\
\frac{\partial \psi^{n}(k)}{\partial f^{b}(k-1)} & \frac{\partial \psi^{n}(k)}{\partial w^{b}(k-1)}
\end{array}\right]
$$

In the observation model, the landmark object information from the sensor is range, bearing and elevation. The presentation of object information in the Cartesian coordinates is

$$
\begin{aligned}
& z(k)=[\rho \varphi \vartheta]^{T} \\
& =\left[\begin{array}{c}
\sqrt{x_{s}^{2}+y_{s}^{2}+z_{s}^{2}} \\
a \tan \left(y_{s} / x_{s}\right) \\
a \tan \left(z_{s} / \sqrt{x_{s}^{2}+y_{s}^{2}}\right)
\end{array}\right]
\end{aligned}
$$

The transformation of the landmark object sensor frame position to the navigation frame is

$$
Z^{n}(k)=P^{n}+\left(T_{b}^{n} * L_{b}^{n}\right)+\left(T_{b}^{n} * T_{s}^{b} * z(k)\right)
$$

The symbol $\alpha$ refers to the angle between the body and the observation sensor camera. The transfer matrix is

$$
T_{s}^{b}=\left[\begin{array}{ccc}
\operatorname{Cos}(-\alpha) & 0 & -\operatorname{Sin}(-\alpha) \\
0 & 1 & 0 \\
\operatorname{Sin}(-\alpha) & 0 & \operatorname{Cos}(-\alpha)
\end{array}\right]
$$


The Jacobian matrix $\nabla h(k)$ used for model linearization in the A-SLAM that is composed of partial differentials of position, velocity and the Euler angle of observation model is

$$
\nabla h=H=\left[\begin{array}{lll}
\frac{\partial \rho(k)}{\partial P^{n}(k)} & \frac{\partial \rho(k)}{\partial V^{n}(k)} & \frac{\partial \rho(k)}{\partial \Psi^{n}(k)} \\
\frac{\partial \varphi(k)}{\partial P^{n}(k)} & \frac{\partial \varphi(k)}{\partial V^{n}(k)} & \frac{\partial \varphi(k)}{\partial \Psi^{n}(k)} \\
\frac{\partial \vartheta(k)}{\partial P^{n}(k)} & \frac{\partial \vartheta(k)}{\partial V^{n}(k)} & \frac{\partial \vartheta(k)}{\partial \Psi^{n}(k)}
\end{array}\right]
$$

In explicit form it is

$$
\begin{aligned}
& \nabla h=\left[\begin{array}{ccc}
\frac{x_{s}}{\sqrt{x_{s}{ }^{2}+y_{s}{ }^{2}+z_{s}^{2}}} & \frac{y_{s}}{\sqrt{x_{s}{ }^{2}+y_{s}{ }^{2}+z_{s}^{2}}} & \frac{z_{s}}{\sqrt{x_{s}{ }^{2}+y_{s}{ }^{2}+z_{s}^{2}}} \\
\frac{-y_{s}}{x_{s}^{2}+y_{s}{ }^{2}} & \frac{x_{s}}{x_{s}{ }^{2}+y_{s}{ }^{2}} & 0 \\
\frac{-x_{s} z_{s}}{\left(x_{s}{ }^{2}+y_{s}{ }^{2}+z_{s}{ }^{2}\right) * \sqrt{x_{s}{ }^{2}+y_{s}^{2}}} & \frac{-y_{s} * z_{s}}{\left(x_{s}{ }^{2}+y_{s}{ }^{2}+z_{s}{ }^{2}\right) * \sqrt{x_{s}{ }^{2}+y_{s}{ }^{2}}} & \frac{\sqrt{x_{s}{ }^{2}+y_{s}{ }^{2}}}{x_{s}{ }^{2}+y_{s}{ }^{2}+z_{s}{ }^{2}}
\end{array}\right]
\end{aligned}
$$

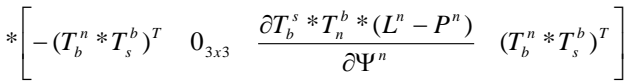

where

$$
\frac{\partial T_{b}^{s} * T_{n}^{b} *\left(L^{n}-P^{n}\right)}{\partial \Psi^{n}}=\left[\begin{array}{lll}
\frac{\partial x_{s}}{\partial \phi} & \frac{\partial x_{s}}{\partial \theta} & \frac{\partial x_{s}}{\partial \psi} \\
\frac{\partial y_{s}}{\partial \phi} & \frac{\partial y_{s}}{\partial \theta} & \frac{\partial y_{s}}{\partial \psi} \\
\frac{\partial z_{s}}{\partial \phi} & \frac{\partial z_{s}}{\partial \theta} & \frac{\partial z_{s}}{\partial \psi}
\end{array}\right]
$$

\section{The Inconsistency Problem and Symptoms}

The EKF structure is developed to apply in non-linear systems. Jocabian matrices are used to linearize the non-linear systems. Using Jacobian matrices causes new errors in the Kalman filter estimations.

Since the EKF is also applicable in non-linear systems, it is used in the SLAM structure. The simultaneous reduction in the error and filter covariance as in the Kalman filter is expected result of the EKF based A-SLAM structure.

Y. Bar-Shalom et al. [18] stated that a state estimator is consistent in the event that it satisfies the following requirements:

- Estimation error mean is zero,

- Real covariance is less than or equal to the covariance calculated by the filter. 
The EKF SLAM filter consistency is analyzed by T. Bailey et al. [9], and it is stated that EFK SLAM is inconsistent in case of the observation of the following symptoms:

- Excessive information gain (estimated error covariance is less than the real covariance),

- Jagged in vehicle movement curve.

\section{Numerical Result}

The state equation given in section 3 and the A-SLAM structure is used in the simulation. The UAV trajectory and landmark is specified due to a certain scenario. The EKF-based A-SLAM simulation results are transferred to the graphics and, using the results filter consistency, analysis is performed.

\subsection{EKF-based A-SLAM}

In the simulation, a UAV at a constant velocity and constant altitude is flying at $30 \mathrm{~m} / \mathrm{sec}$ over an area of $1400 \mathrm{~m} \times 1400 \mathrm{~m}$ in a closed loop. The landmark detection with camera and position determination is performed. The UAV is supposed to start flying at $[0,0,0]$. The total simulation time is bounded at 240 seconds. An IMU is used as a sensor. The eighth landmark object is depicted in the map. The UAV trajectory and landmarks are depicted in Fig. 1.

The UAV directional velocity in Fig. 2 is given versus time. The UAV localization error is shown in Fig. 3, the directional velocity error in Fig. 4, the Euler angle error in Fig. 5, and the $\mathrm{X}$ axis position error and filter covariance in Fig. 6.

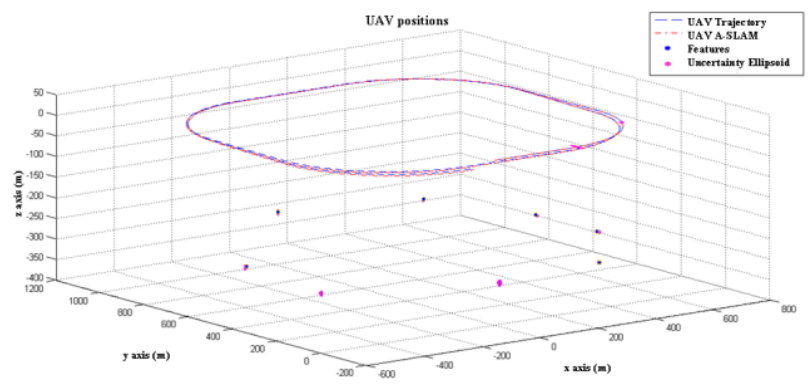

Figure 1

UAV and Landmark Localization. In this plot, the blue dashed line is the UAV trajectory, the red dotted line is the A-SLAM, the blue ellipse is the landmark localization, and the red ellipse is the uncertainty ellipsoid 


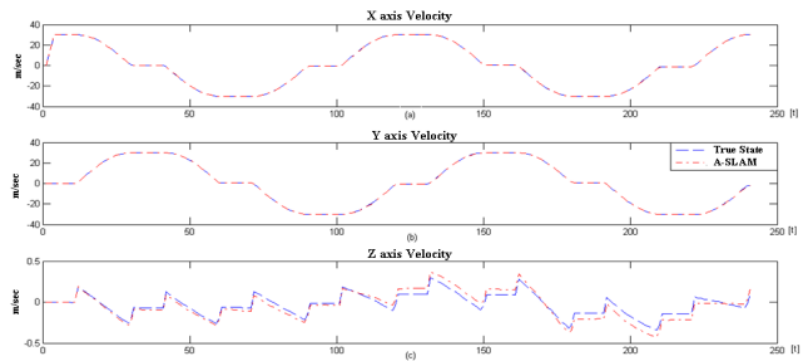

Figure 2

UAV Directional Velocities versus Time. (a) X axis velocity, (b) Y axis velocity, (c) Z axis velocity. In this plot, the blue dashed line is the UAV real velocity, and the red dotted line is the velocity calculated by A-SLAM

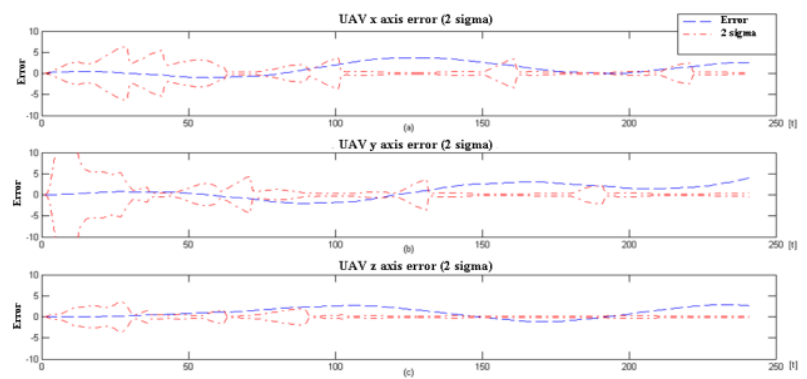

Figure 2

UAV Localization Error. (a) UAV X axis error, (b) UAV Y axis error, (c) UAV Z axis error. In this plot, the blue dashed line is the UAV position error, and the red dotted line is the 2 sigma confidence interval
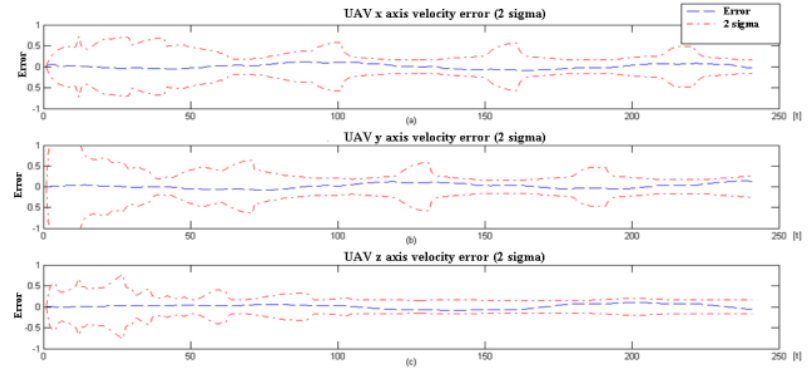

Figure 3

UAV Velocity Error. (a) UAV X axis velocity error, (b) UAV Y axis velocity error, (c) UAV Z axis velocity error. In this plot, the blue dashed line is the UAV velocity error, and the red dotted line is the 2 sigma confidence interval 

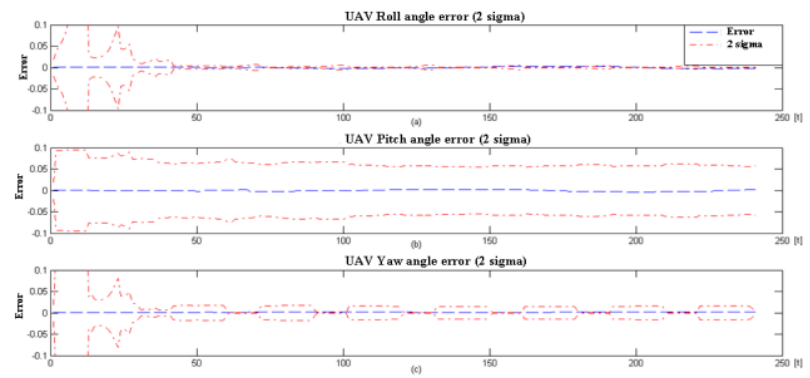

Figure 4

UAV Euler Angle Error. (a) UAV roll angle error, (b) UAV pitch angle error, (c) UAV yaw angle error. In this plot, the blue dashed line is the UAV angle error, and the red dotted line is the 2 sigma confidence interval

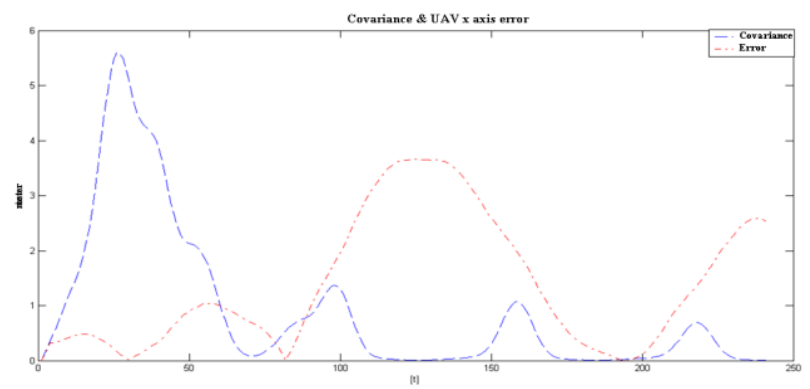

Figure 5

Position Error on X Axis and Filter Coveriance. In this plot, the blue dashed line is the filter X axis covariance, and the red dotted line is the $\mathrm{X}$ axis error

\subsection{First Symptom: Information Gain}

It is necessary that the filter covariance should decrease over time in a consistent filter structure, and the error must be smaller than the estimated covariance. In a similar manner, the consistency of the EKF based A-SLAM structure can be analyzed.

This analysis can be done in two different ways: first by checking the error and covariance relation by using simulation results, and second, by making use of the normalize estimation error square value. 


\subsubsection{Filter Covariance - Error Relation}

According to the values used in the simulation scenario, the second moments of the UAV's $[\mathrm{x}, \mathrm{y}, \mathrm{z}]$ position variables with the Airborne SLAM inspection of the estimated values of covariance are:

As the second moment:

$P(k) \Rightarrow \operatorname{var}(x)=0,1628 ; \operatorname{var}(y)=0,6432 ; \operatorname{var}(z)=0,1751$.

The estimated Coveriance:

$$
P(k \mid k) \Rightarrow P_{x}=0,0051 \quad P_{y}=0,0258 \quad P_{z}=0,0063
$$

Since $P(k \mid k)<P(k)$ the filter is inconsistent.

\subsubsection{The Normalized Estimation Error Square}

If the actual statistical values are unknown but the true state $x(k)$ is known, the normalised Estimation Error Squared (NEES) can be used.

Let $\mathrm{N}$-dimensional random numbers vector $\mathrm{x}$ be in Gaussian distribution, the mean be $\bar{x}$ and covariance be $\mathrm{P}$, then $\mathrm{q}=(x-\bar{x})^{\prime} \cdot P^{-1} \cdot(x-\bar{x})$ can be written in chisquare distributed quadratic form with $\mathrm{N}$ dimension of freedom. The Chi-square distribution can be defined as the ratio of the squares of two Gaussian distributed variables.

The chi-square conformity test is a decision as to whether the difference between the expected and obtained value is within a specified limit or not. The process test not only variable distribution but also the whole filter system.

In the EKF-based A-SLAM structure, if the UAV's real position $x(k)$ is known, then the difference between the UAV's real position $x(k)$ and calculated position, the term $x(k \mid k)$ can be computed. The NEES or filter performance characteristic is:

$$
\varepsilon(k)=(x(k)-x(k \mid k))^{\prime} \cdot P(k \mid k)^{-1} \cdot(x(k)-x(k \mid k))
$$

The NEES distribution is expected to be a chi square, or if the computed distribution is not chi-square, then the filter is not consistent.

The chi-square distribution depends on the degrees of freedom of system. The expected bounds of distribution with degrees of freedom are determined using a table. The UAV's position is specified by nine degrees of a freedom-position vector composed of the velocity and angle values. The NEES, with nine degrees of freedom and its reliability boundary, is given in Fig.6. The computed NEES in a filter should be within the reliability boundary for consistency. Since it is not, the proposed filter of the EKF based A-SLAM is inconsistent. 


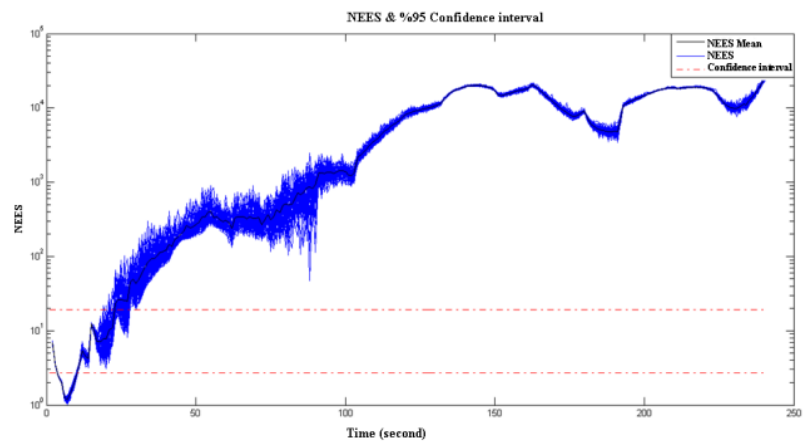

Figure 6

EKF Based A-SLAM, 50 Monte Carlo Simulation, NEES 95\% confidence interval. In this plot, the blue line is the NEES, the black line is the NEES mean, and the red dashed lines are the $95 \%$ confidence interval

\subsection{Second Symptom: Jagged UAV Trajectory}

The other results of the filter inconsistency are jumps in the UAV's trajectory. That originates from the UAV's correction when it detects a landmark. This symptom appears by the size of the update, and the UAV's position tends to be much larger than the actual error. When the landmark is observed, the UAV's trajectory is jagged, as shown in Fig. 7.

Both symptoms indicate inconsistency with the A-SLAM consistency investigation. Despite the accumulation of errors, which are the difference between the UAV's real position and the filter estimation, the A-SLAM covariance decreases, as shown in study. The reason for filter inconsistency is the limited observability of the A-SLAM or other observability problems.

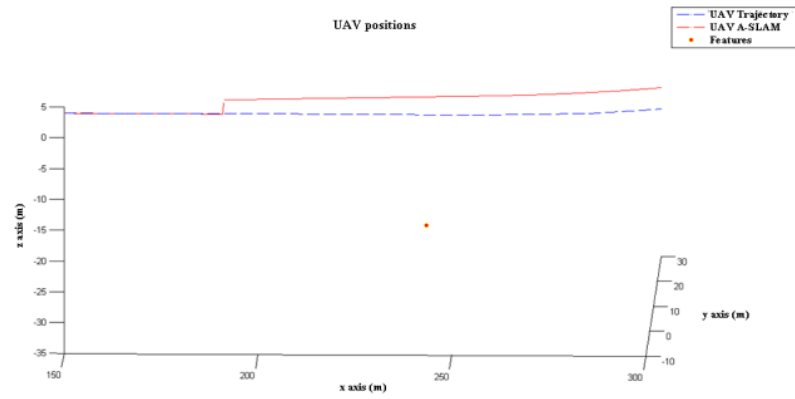

Figure 7

Jagged UAV Trajectory. In this plot, the blue dashed line is the UAV trajectory, the red line is the ASLAM, and the red ellipse is the landmark localization 


\section{Conclusion and Future Work}

In this paper, a mathematical model and the simulation results for the EKF-based A-SLAM structure of UAVs are presented in order to investigate the consistency of the EKF-based A-SLAM. It is observed that the error stems from the Jacobian matrices used for linearization. Therefore, the uncertainty emerged from Jacobian matrices and the effects of the landmark uncertainty that causes error accumulation. The filter estimation covariance should be greater or equal to the error, and the consistent filter is supposed to calculate covariance in accordance with error. Since it is stated that the filter is not able to respond to error accumulation, this implies filter inconsistency.

The inconsistency in the EKF-based A-SLAM structure was emphasized and background information for the solution of the problem was provided. It was also observed that main reason for filter inconsistency is the information gain arising from unobservable subspace. The imperfect or incorrect information coming from the unobservable subspace filter does not respond to error accumulation.

In future works, methods such as extending observability, designing an observability constrained filter, or constructing a full observable filter structure can be applied to making the filter consistent. Furthermore, Jacobian matrices that are used in the A-SLAM, can be calculated by methods, except for differential methods.

\section{References}

[1] R. Smith and P. Cheesman, On the Representation of Spatial Uncertainty, Int. J. Robot. Res., Vol. 5, No. 4, pp. 56-68, 1987

[2] G. Dissanayake, H. F. Durrant-Whyte and T. Bailey, A Computationally Efficient Solution to the Simultaneous Localisation and Map Building (SLAM) Problem, ICRA, 2000

[3] T. Bailey and H. F. Durrant-Whyte, Simultaneous Localization and Mapping (SLAM): Part I The Essential Algorithms., IEEE Robotics and Automation Magazine, 2006

[4] T. Bailey and H. F. Durrant-Whyte, Simultaneous Localisation and Mapping (SLAM): Part II State of the Art., IEEE Robotics and Automation Magazine, September 2006

[5] J. Andrade-Cetto, and A. Sanfeliu, The Effects of Partial Observability in SLAM, IEEE Int. Conf. Robot. Automat., New Orleans, pp. 397-402, 2004

[6] M. Bryson, and S. Sukkarieh, Observability Analysis and Active Control for Airborne SLAM, IEEE Transaction on Aerospace and Electronic Systems, Vol. 44, No. 4, pp. 261-280, 2008

[7] G. P. Huang, N. Trawny, A. I. Mourikis and S. I. Roumeliotis, Observability-based Consistent EKF Estimators for Multi-Robot 
Cooperative Localization, Autonomous Robots, Volume 30, Number 1, pp. 99-122, 2011

[8] T. Vidal-Calleja, J. Andrade-Cetto and A. Sanfeliu, Conditional for Suboptimal Filter Stability in SLAM, IEEE Inernational Conferance on Intelligent Robots and Systems, Sendai Japan, 2004

[9] T. Bailey, J. Nieto, J. Guivant, M. Stevens and E. Nebot, Consistency of the EKF-SLAM Algorithm, IROS'06 IEEE/RSJ International Conference on Intelligent Robots and Systems, Beijing, China, 2006

[10] J. A. Castellanos, J. Neira, J. Tardos, Limits to The Consistency of EKFBased SLAM, IAV2004 $5^{\text {th }}$ IFAC Symp. on Intelligent Autonomous Vehicles, 2004

[11] J. Kim and S. Sukkarieh, Airborne Simultaneous Localisation and Map Building, IEEE Int. Conf. Robot. Automat., Taipei Taiwan, 2003

[12] A. E. Oguz and H. Temeltas, Extended Kalman Filter Based Airborne Simultaneous Localization And Mapping, Journal of Aeronautics and Space Technologies, Vol. 6, No. 2, pp. 69-74, 2013

[13] J. Kim, S. Sukkarieh, S. Wishart, Real-Time Navigation, Guidance and Control of a UAV Using Low-Cost Sensors, International Conferance of Field and Service Robotics, Yamanashi, Japan, pp. 95-100, 2003

[14] J. Kim, S. Sukkarieh, Autonomous Airborne Navigation in Unknown Terrain Environments, IEEE Transaction on Aerospace and Electronic Systems, Vol. 40, No. 3, pp. 1031-1044, 2004

[15] S. Kurnaz, O. Cetin and O. Kaynak, Fuzzy Logic Based Approach to Design Flight Control and Navigation Tasks for Autonomous UAVs, Journal of Intelligent and Robotic Systems, v. 54, pp. 229-244, 2009

[16] S. Kurnaz, and O. Cetin, Autonomous Navigation and Landing Tasks for Fixed Wing Small Unmanned Aerial Vehicles, Acta Polytechnica Hungarica, Vol. 7, No. 1, 2010

[17] L. M. Paz, and J. Neira, Optimal local map size for GKF-based SLAM, IROS'06 IEEE/RSJ International Conference on Intelligent Robots and Systems, Beijing, China, 2006

[18] Y. Bar-Shalom, X. Rong Li and T. Kirubarajan, "Estimation with Applications to Tracking and Navigation", John Wiley and Sons, 2001, pp. 232-145 